Musées, Patrimoine et Culture scientifiques et techniques

$138 \mid 2011$

novembre - décembre 2011

\title{
La désinsectisation des biens patrimoniaux par la chaleur
}

Pest control on museum objects using heat-treatment

\section{Michèle Gunn}

\section{OpenEdition \\ Journals}

Édition électronique

URL : http://journals.openedition.org/ocim/992

DOI : 10.4000/ocim.992

ISSN : 2108-646X

Éditeur

OCIM

Édition imprimée

Date de publication : 1 novembre 2011

Pagination : 41-47

ISSN : 0994-1908

Référence électronique

Michèle Gunn, «La désinsectisation des biens patrimoniaux par la chaleur », La Lettre de l'OCIM [En ligne], 138 | 2011, mis en ligne le 01 novembre 2013, consulté le 30 avril 2019. URL : http:// journals.openedition.org/ocim/992 ; DOI : 10.4000/ocim.992 


\section{La désinsectisation des biens patrimoniaux par la chaleur}

\section{Michèle Gunn *}

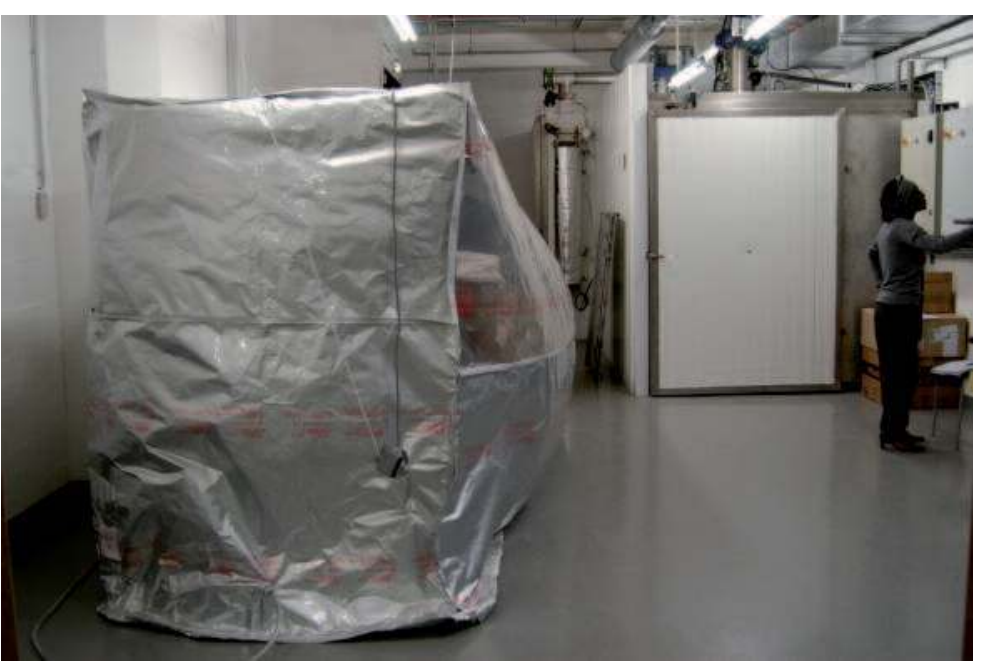

La salle de désinsectisation au musée du quai Branly () M. Gunn
Bien que largement répandue au Canada et au Royaume-Uni, la désinsectisation des objets patrimoniaux par la chaleur demeure encore assez peu utilisée en France : la présentation des protocoles de cette méthode de son application au musée du quai Branly permet $d^{\prime}$ en mesurer les apports en matière de désinfestation.

Cet article vise à faire connaître une méthode de désinsectisation qui utilise la chaleur, méthode qui n'est pas bien connue en France. Les réserves quant à l'utilisation de cette méthode résident dans l'idée reçue selon laquelle le froid conserve et la chaleur dégrade. Les craintes viennent également du fait que souvent l'impact des variations climatiques sur les matériaux est mal maîtrisé.

Les études menées à l'Institut Canadien de Conservation et au Muséum d'Histoire naturelle de Londres indiquent la faisabilité du traitement par la chaleur quand les conditions sont bien contrôlées. L'application de cette méthode de désinsectisation au musée du quai Branly conforte encore une fois sa faisabilité et son efficacité et le gain de temps face à une infestation inopinée.

\section{Introduction}

Le risque de dégradation du patrimoine par les organismes vivants est un risque récurrent auquel il faut apporter une attention toute particulière. Entre la négligence ou la psychose face à l'infestation, il existe une attitude modérée, un juste milieu basé sur une analyse rationnelle des situations.

Le risque d'infestation des collections est d'autant plus élevé que les collections sont constituées de matériaux organiques
* Michèle Gunn est chef du service ConservationRestauration au Centre des Monuments nationaux agnoko-michelle.gunn@monuments-nationaux.fr 
et de composites complexes. En effet, ces matériaux constituent des cibles pour toute sorte d'insectes qui y puisent nourriture et y trouvent des habitats de nidification.

Pour lutter contre l'infestation, une gamme très large de composés chimiques ont très tôt été utilisés en grande quantité, souvent de façon anarchique et répétée : des produits à base de mercure ou d'arsenic, des gaz tels que le bromure de méthyle ou l'oxyde d'éthylène ou encore des solides chlorés faciles à sublimer comme le lindane (Flieder, F. ; Capderou, C. ; Rocquebert, M.-F. ; Zycherman, L. A. ; Schrock, J.-R.).

Certains de ces composés chimiques composés ont d'abord été utilisés en agroalimentaire ou dans le domaine médical puis ont été adaptés progressivement au domaine patrimonial. Avec le temps beaucoup de ces produits se sont révélés très nocifs pour les êtres vivants et l'environnement et également pour les œuvres. Face à ce désastre, une prise de conscience s'est peu à peu effectuée avec une volonté mondiale d'utiliser plus rationnellement des insecticides dans tous les domaines.

Les années 1980 et 1990 ont connu une révolution dans les mentalités par rapport à la lutte contre l'infestation dans le milieu patrimonial : c'est la naissance du concept de l'IPM, Integrated Pest Management, la gestion intégrée de l'infestation. La lutte contre l'infestation va alors s'intégrer dans une politique globale de conservation du patrimoine (Zycherman, L.-A. ; Schrock, J.-R. ; Pinninger, D. ; Kingsley, H.).

De nos jours, avec l'essor du principe de développement durable, et la place de plus en plus importante de la médecine du travail (maladies professionnelles), ces produits font de plus en plus l'objet de réglementations sévères. Des solutions alternatives sont donc recherchées pour préserver le patrimoine sous tout ses aspects (culturel, vivant, environnement).

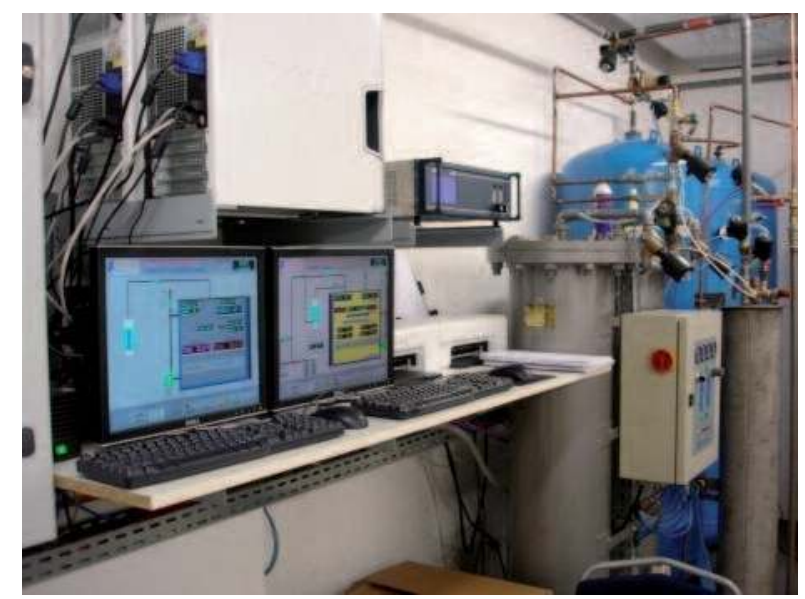

La supervision informatique (c) M. Gunn
Les différents types de désinsectisation peuvent se classer en quatre catégories :

- les traitements chimiques (composés gazeux ou liquides); - les traitements utilisant les ondes électromagnétiques (Rayons gamma et micro-ondes) ;

- les traitements par modification de la température (froid et chaleur) ;

- les traitements en atmosphères modifiées ou contrôlées (anoxie par azote, hélium, argon, ou dioxyde de carbone) (Selwitz, C. ; Maekava, S.).

L'avantage de l'anoxie est son innocuité par rapport aux matériaux et l'inconvénient est la durée longue des traitements par rapport aux autres techniques dont les temps de traitement sont de quelques minutes à une semaine incluant les temps de manipulation.

\section{La technique du traitement par la chaleur}

\section{Dans l'industrie du bois}

Si l'utilisation de la chaleur est très peu connue dans le domaine patrimonial en France, le procédé est largement répandu dans l'industrie du bois dans le cadre des mesures phytosanitaires. Ainsi, la Norme internationale pour les Mesures phytosanitaire $n^{\circ} 15$ (NIMP15) stipule pour la marque $\mathrm{HT}^{(1)}$ que «le matériau d'emballage en bois doit être chauffé selon une matrice temps/température précise permettant d'assurer une température minimale de $56^{\circ} \mathrm{C}$ pendant une durée ininterrompue d'au moins 30 minutes dans l'ensemble du bois (y compris en son cœur) : séchage à l'étuve, l'imprégnation chimique sous pression favorisée par la chaleur, micro-ondes » (Site Internet NIMP15).

Ainsi, le bois brut est traité dans des fours pouvant atteindre des températures de $200^{\circ} \mathrm{C}$, avec ou sans vapeur d'eau avec pour objectif de produire du bois de qualité durable sans adjonction de produits chimiques. Cette technique a été ensuite adaptée au milieu du patrimoine, avec une attention particulière à la stabilisation de l'hygrométrie.

\section{En milieu patrimonial}

Les données bibliographiques donnent des températures de traitement comprises entre $50^{\circ} \mathrm{C}$ et $55^{\circ} \mathrm{C}$ pour une humidité relative comprise entre $50 \%$ et $60 \%$ et une durée d'opération de 24 à 48 heures incluant la montée et la descente en température avec un temps de traitement de 3 heures. À cette température la mort est instantanée pour la plupart des insectes (Ackery, P. et al. ; Strang, T. ; Kigawa, R.).

\section{Les études préalables pour adapter le traitement thermique industriel au patrimoine}

L'Institut Canadien de Conservation a, dès les années 1990, exploré la faisabilité du traitement thermique sur les biens (Strang, T.). 
La société Thermo Lignum ${ }^{\circledR}$ basée en Allemagne, en Autriche et en Grande-Bretagne a développé depuis 1990 un système de traitement thermique, Warmair, avec une régulation fine de l'hygrométrie assistée par ordinateur. Cette société possède des unités fixes et des unités mobiles de traitement par la chaleur et par anoxie et assure ainsi des prestations à travers l'Europe (Thermo Lignum, site Internet).

Le Muséum d'Histoire naturelle de Londres a également exploré la possibilité d'appliquer le traitement thermique sur ses collections, en particulier la collection d'entomologie, en collaboration avec Thermo Lignum ${ }^{\circledR}$ (Ackery, P. et al.).

\section{Études menées à l'Institut} Canadien de Conservation

Ces études visent à explorer l'impact de l'élévation de la température sur les propriétés biologiques et physico-chimiques des matériaux et à établir si oui ou non un tel traitement peut être envisagé sur des collections patrimoniales sans risques majeurs de détérioration. En effet, sous des conditions de températures élevées, les risques de dégradation peuvent être de nature biologique, chimique ou mécanique. Les tests ont porté sur les aspects suivants : changements dimensionnels liés à la sorption et désorption de vapeur d'eau, l'expansion thermique, l'impact sur la germination des graines et l'aptitude à éradiquer les insectes à tous les stades de développement.

L'auteur, Tom Strang, indique par ailleurs que le traitement par la chaleur a très tôt été pensé pour la désinsectisation des collections. En effet dans une lettre au président et aux membres de la Royal Society en 1771 sur la préservation des oiseaux naturalisés S.-T. Kuckahn vente les mérites du traitement par la chaleur dont l'avantage est son efficacité à éradiquer également les œufs des insectes, stade de développement le plus résistant.

\section{Effet sur la teneur en eau des matériaux}

La teneur en eau d'un matériau, par exemple le bois, est définie comme la différence entre la masse du matériau humide et la masse du matériau sec divisé par la masse du matériau sec. Cette teneur en eau dépend de la nature du matériau. Pour le même matériau, la teneur en eau peut varier en fonction du climat environnant. À un taux d'humidité relative donné, la teneur en eau d'un matériau est appelée le taux d'humidité à l'équilibre (THE). Celui-ci varie lors d'échanges de vapeur d'eau entre le matériau et son environnement. Cet échange se produit lorsque la différence de pression partielle entre les deux milieux est différente. La variation du THE induit des variations dimensionnelles du matériau (voir schéma ci-dessus).

En s'appuyant sur des courbes isothermes d'absorption de vapeur d'eau de différents matériaux, il est noté que pour une humidité relative ambiante donnée, le THE varie en

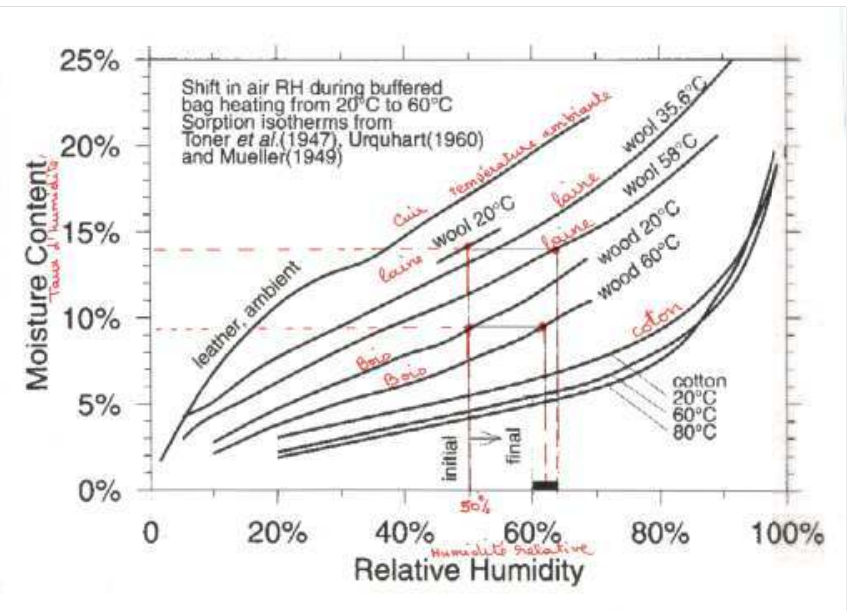

Variation du THE en fonction de la température et de l'humidité relative et des matériaux

(d'après Strang T.).

fonction de la température : lorsque la température augmente, le THE diminue et inversement. Cela peut s'expliquer par l'agitation des molécules d'eau du matériau, agitation thermique qui contraint l'absorption de la vapeur d'eau ambiante. De ce fait pour maintenir le THE constant lorsque la température augmente, il faut augmenter l'humidité relative ambiante par un apport d'eau. Dans le cas contraire, le matériau s'appauvrit en humidité.

Une façon de contourner cet apport d'eau est de travailler en système étanche en emballant les objets dans des bulles coupe-vapeur, dans des sacs en polyéthylène par exemple. L'utilisation de matériaux tampons, comme le coton, est également une autre alternative. Dans un système étanche, l'eau libérée par les objets lors de l'élévation de la température reste enfermée dans le système et contribue ainsi à maintenir l'humidité relative ambiante constante. Il se produit un équilibre entre l'humidité relative ambiante et le taux d'humidité des objets. Cet effet, qualifié d'effet tampon de l'humidité, permet de réguler plus facilement l'hygrométrie sans la contrainte de devoir injecter de la vapeur d'eau lorsque la température augmente.

Il découle de ces études que la densité de chargement idéal pour le matériau bois serait de $100 \mathrm{~kg}$ par $\mathrm{m}^{3}$, pour des objets en bois ayant séjourné à $20^{\circ} \mathrm{C}$ et $50 \%$ d'humidité relative. Ainsi la variation du THE ne sera que de $1 \%$ lorsque la température passera de $20^{\circ} \mathrm{C}$ à $60^{\circ} \mathrm{C}$ et l'humidité relative de $50 \%$ à $63 \%$.

La même variation de $1 \%$ du THE se produit lorsque l'humidité relative ambiante varie de 5-10\%. Dans les recommandations ASHRAE (2), une telle fluctuation correspond à un climat du type $\mathrm{AA}$ à $\mathrm{A}$, soit un climat qui n'induit qu'un minimum de risques de dommages mécaniques pour les objets les plus vulnérables (Michalsky, M.). 


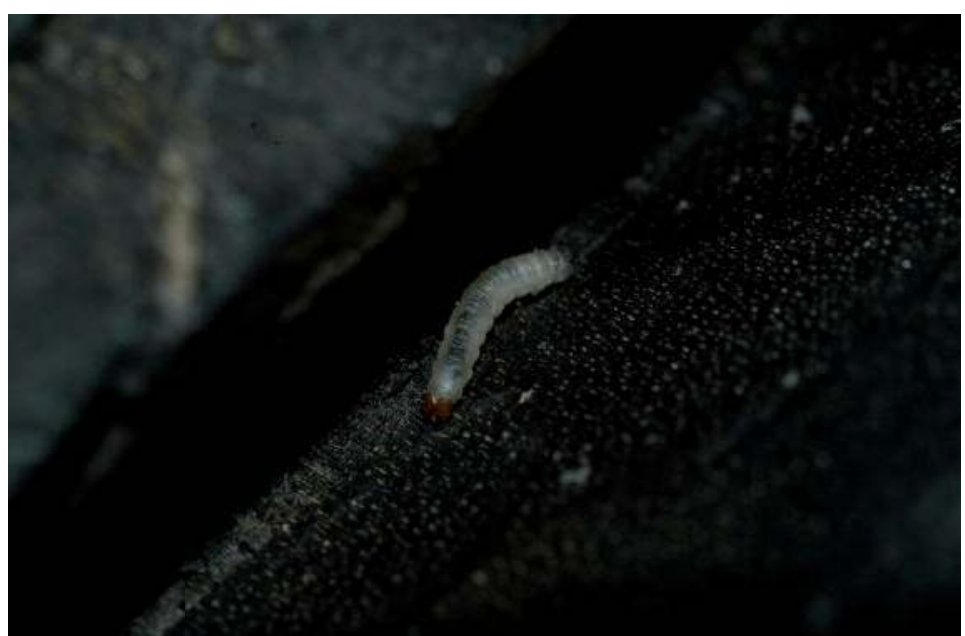

Larve de mite vivante (c) M. Gunn

\section{Les risques de dommages biologiques}

Un système clos avec des températures élevées et une humidité relative avoisinant les $60 \%$ peut faire craindre une prolifération de moisissures. Cependant, la durée très courte du traitement thermique ne peut favoriser une telle dégradation. De même, des graines ont pu germer après qu'elles aient été soumises au traitement thermique, montrant ainsi que l'élévation de la température n'a pas dénaturé leur patrimoine génétique.

\section{Études menées par Thermo Lignum ${ }^{\circledR}$}

Des études menées entre autres dans le cadre d'une thèse sur différents matériaux, par exemple des panneaux de bois peints et/ou vernis et des peaux soumis au traitement thermique par la méthode Thermo Lignum ${ }^{\circledR}$ n'ont pas montré de signes de changements de propriétés mécaniques ni de variations dimensionnelles ni de changement de $\mathrm{pH}$. Un tel traitement est cependant déconseillé pour les peaux dégradées (Thomson, R.-S. ; Thermo Lignum ${ }^{\circledR}$, mémoire de diplôme de Fachhochschule).

\section{Étude au Muséum d'Histoire naturelle de Londres}

L'objectif était de voir si les collections d'entomologie pouvaient subir sans dommages le traitement par la chaleur avec le procédé Thermo Lignum ${ }^{\circledR}$, en les conservant dans les armoires de rangement et en évitant l'emballage dans les bulles plastiques coupe-vapeur comme préconisé par l'ICC. En effet, l'emballage est une étape de manipulation supplémentaire qui peut s'avérer vite contraignante et coûteuse pour la désinsectisation de masse avec un rallongement de la durée totale du traitement.

Les divers agencements des chargements effectués dans le cadre de cette étude ont montré qu'une circulation d'air de $5 \mathrm{~cm}$ autour des armoires était nécessaire à la bonne répartition et diffusion de la chaleur. Les enregistrements de température et d'hygrométrie tout au long du cycle ont indiqué une bonne stabilité de l'humidité relative. L'extraction et l'identification d'ADN avant et après action de la chaleur n'ont montré aucun changement. Ce résultat est en accord avec ceux obtenus pour la germination des graines (Ackery, P. ; Testa, J.-M. et al.).

\section{Le système installé au musée du quai Branly}

Dans le souci d'être toujours très réactif face aux problèmes d'infestation, tout en respectant son engagement pour le développement durable, le musée du quai Branly, qui s'est beaucoup investi dans l'anoxie (Gunn, M.), a souhaité diversifier ses méthodes de lutte contre l'infestation. Pour rester fidèle aux techniques ne faisant pas appel à des gaz toxiques, les techniques qui correspondent à ce critère sont les traitements par le froid, la chaleur, les micro-ondes, les rayons gamma.

\section{Description de l'installation}

L'installation comprend une enceinte en panneaux sandwich d'environ $12 \mathrm{~m}^{3}$ pouvant recevoir 4 palettes standard. La machinerie comprend un système de 4 ventilateurs dont 1 pour le chauffage et 3 à l'intérieur de l'enceinte pour assurer l'homogénéisation de l'atmosphère, un système de batterie pour le chauffage et une station de vapeur pour la régulation de l'hygrométrie. Une série de capteurs de température et d'hygrométrie numérotés de 1 à 6 , fixes ou mobiles, est répartie à l'intérieur de l'enceinte de façon à permettre d'apprécier l'homogénéité du climat de l'enceinte.

Les différentes phases du traitement sont gérées par un automate. Une supervision informatique permet de paramétrer la température, l'hygrométrie et la durée de traitement via une série d'écrans synopsis. À la fin du traitement, rapport et courbe retraçant l'historique des conditions climatiques sont émis (voir schéma ci-contre).

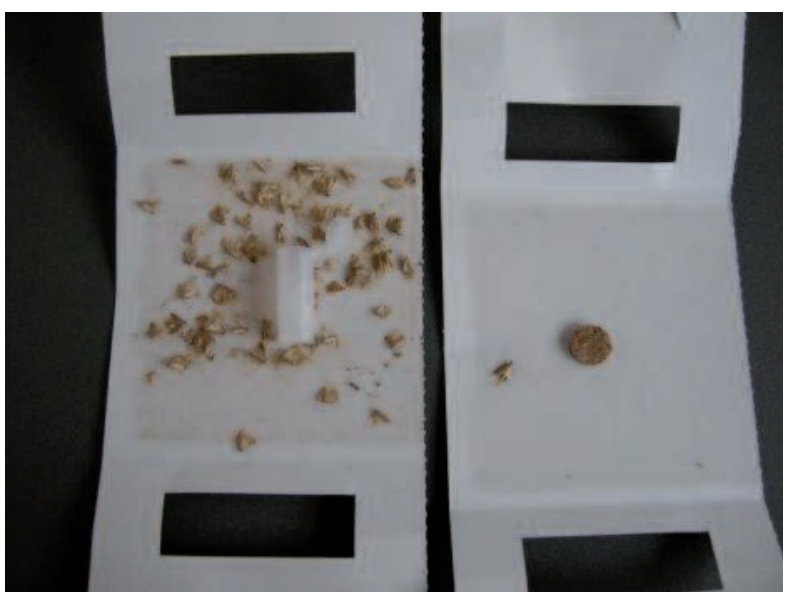

Mise en évidence de la spécificité des phéromones. Les deux pièges ont été mis côte à côte. Seul le piège contenant la phéromone pour mite de vêtements s'est révélé efficace. L'autre phéromone est ciblé mite alimentaire. (c) M. Gunn 


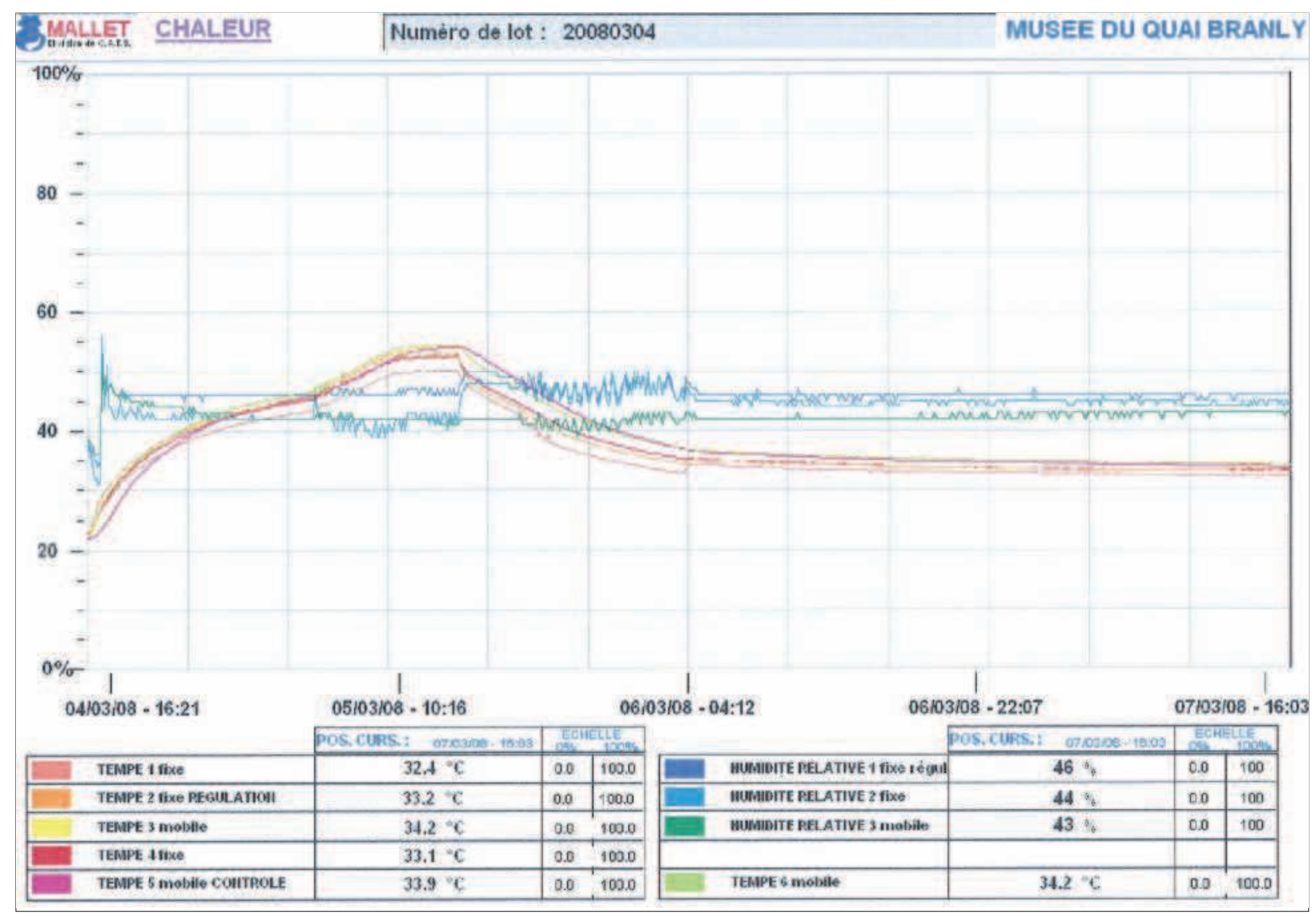

Conditions climatiques au cours du traitement, température et humidité relative (T1 H1 : sonde de température et d'hygrométrie fixe ; $\mathrm{T} 1$ : pour la régulation de la température. ; $\mathrm{T} 2 \mathrm{H} 2$ : sonde de température et d'hygrométriefixe; $\mathrm{H} 2$ : pour la régulation de l'hygrométrie ; $\mathrm{T} 3 \mathrm{H} 3$ : sonde de température et d'hygrométrie mobile à mettre au sein du chargement pour suivre les conditions au sein du chargement ; T4 : sonde de température fixe ; T5 : sonde de température mobile à mettre dans le matériau de référence, pour le contrôle ; T6 : sonde de température mobile à mettre au sein du changement).

\section{Mise en place des conditions opératoires}

L'enceinte a d'abord été testée vide d'objets puis, chargée de palettes en bois. Une série d'objets non patrimoniaux en fibres végétales, en bois et en peau provenant de l'atelier pédagogique, a été soumise à ce traitement. Après plusieurs essais de programmation de la montée et de la descente en température, les conditions opératoires adéquates ont été obtenues. La durée du traitement a été adaptée selon les résultats décrits dans la bibliographie.

Les objets ne sont pas emballés dans des bulles coupe-vapeur mais dans du papier de soie et posés sur des palettes en bois. Le protocole suivant a été adopté : $0,1^{\circ} \mathrm{C}$ par minute pour la montée et la descente en température et un maintien de la température de traitement soit $52^{\circ} \mathrm{C}$ pendant 3 à $4 \mathrm{~h}$. Le retour aux conditions climatiques initiales dépend du climat de la salle. En effet, à la fin du traitement, le refroidissement de l'enceinte s'opère par mélanges successifs et progressifs de l'air de l'enceinte et de l'air de la salle par aspiration de l'air de la salle. Cette phase implique la non étanchéité totale de l'enceinte.

\section{Test sur des insectes}

\section{Sur des larves de mites}

Des larves de mites (Tineola bisselliella) ont été soumises au traitement thermique. La mort des insectes procède par dessiccation comme le montre la photographie ci-contre.

\section{Sur des larves et des œufs de capricornes des maisons (Hylotrupes bajulus)}

Cet insecte a été choisi pour sa disponibilité. En effet celuici est en élevage au CFBA (3). Trois expérimentations dans les mêmes conditions opératoires $\left(52^{\circ} \mathrm{C}, 4\right.$ heures $)$ ont été menées. Chaque expérimentation comportait 4 boîtes de larves et 4 boîtes d'œufs dont 3 de chaque ont été introduites dans l'enceinte et 1 de chaque laissée dans la salle comme témoin. Chaque boîte était constituée de 5 blochets infestés de larves âgées d'environ 14 semaines et d'œufs de moins d'une semaine.

À la fin de l'expérimentation les échantillons sont renvoyés au CFBA pour être remis en étuve afin d'établir le taux de mortalité. L'ensemble des trois expérimentations a nécessité 60 blochets de larves et 60 blochets d'œufs. Dans tous les cas le résultat a été de $100 \%$ de mortalité. 
Gestion d'infestations avec le traitement thermique

\section{Le cas des objets de l'atelier pédagogique}

L'atelier pédagogique du musée possède une large gamme d'instruments de musique (balafons, coras, sanzas...), des calebasses et des nattes en fibres végétales pour asseoir petits et grands. Cette collection non patrimoniale est achetée directement chez des fournisseurs. Une infestation par des insectes à larves xylophages s'y était déclarée. Le service conservation préventive a été sollicité pour mettre en place un protocole pour un meilleur suivi sanitaire Tous les objets ont été traités soit par anoxie soit par la chaleur en fonction de l'urgence de la restitution à l'atelier.

\section{Le cas des tapis exposés sur le parcours de visite}

La présence récurrente de mites des vêtements dans les pièges localisés principalement sur le parcours de visite, dans la zone Amérique, à proximité de vitrines contenant de la laine et des plumes et dans la boîte muséographique contenant des tapis en zone Afrique a conduit à prendre des mesures sanitaires rapides pour empêcher la prolifération de l'infestation dans tous les espaces du musée. Aussi, afin de ne pas priver les visiteurs de ces œuvres sur une longue période, le choix a été fait d'un traitement des tapis par la chaleur. Parallèlement pour assainir l'espace du musée, une diffusion d'insecticides hydro-réactifs, le Dobol ${ }^{\circledR}$, a été effectuée par une société 3D (dératisation, désinsectisation, désinfection), suivie d'une action systématique de nettoyage du sous-plancher par une société spécialisée ${ }^{(4)}$.

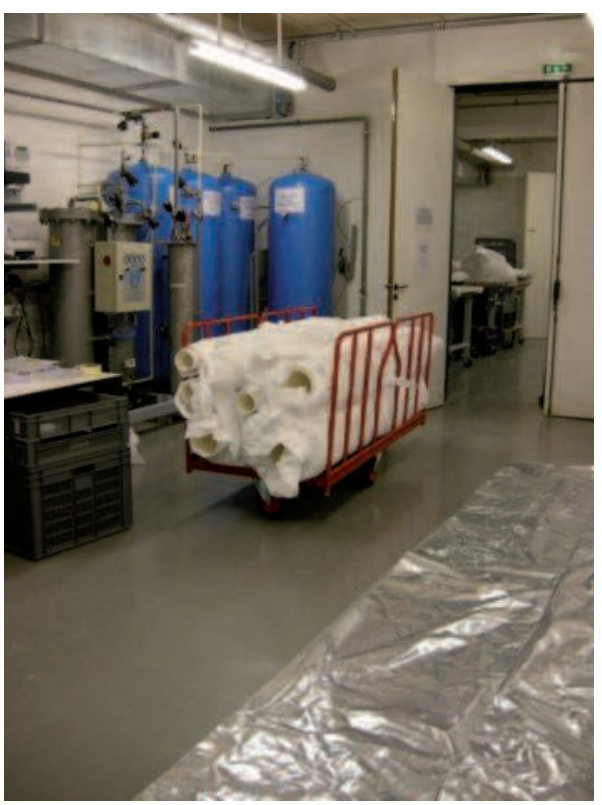

Vue du conditionnement de tous les tapis dans la salle de désinsectisation (c) M Gunn

\section{Conclusion}

Ces études ont montré que le traitement par la chaleur est efficace sur un large spectre d'insectes y compris ceux couramment rencontrés en milieu patrimonial. Les collections patrimoniales peuvent ainsi être désinsectisées efficacement par la méthode de la chaleur. L'isolation des biens dans des films coupe-vapeur ou l'utilisation de matériaux tampons permet de mieux contrôler ou de stabiliser l'hygrométrie ambiante et d'éviter ainsi l'assèchement des matériaux. Lorsque le contrôle de l'hygrométrie est bien maîtrisé, les risques de dégradation liés à l'élévation de la température restent minimes sur les matériaux. Il faut toutefois être prudent pour les matériaux fragiles comme des peaux usées ou pour les matériaux pouvant contenir des corps gras ou tout autre matériau à bas point de fusion.

L'auteur tient à adresser ses remerciements à ses supérieurs hiérarchiques pour la confiance que ceux-ci lui ont témoigné en lui confiant ce projet, à ses collègues du pôle Régie et du pôle Conservation-Restauration pour leur collaboration pour faire aboutir ce projet et à la direction des Moyens Techniques et de Sécurité du musée pour son aide effective sur les questions techniques.

\section{Notes}

(1) Marque HT apposée sur les emballages en bois pour Heat Treatment (traitement par la chaleur).

(2) American Society of Heating Refrigerating and Air-Conditioning Engineers

(3) Institut Technologique Foret Cellulose, Bois-construction Ameublement

(4) Cette opération, longue et délicate, est toujours en cours. Elle est supervisée par la Direction des Moyens Techniques et de Sécurité du musée.

\section{Bibliographie}

Ackery, P., Pinninger, D., Doyle, A. et Roux, K. Heat treatment of entomological drawers using the Thermo Lignum ${ }^{\circledR}$ Heat Process, Collection Forum, 2005, vol. 19 (1-2), pp. 15-22.

Ackery, P., Testa, J.-M., Ready, A.-M., Doyle, A. et Pinninger, D. Effects of high temperature pest eradication on DNA in Entomological collections, Studies in conservation, 2004, vol. 49, pp. 35-40.

Flieder, F. et Capderou, C. Sawvegarde des collections du Patrimoine, la lutte contre les détériorations biologiques. Paris : CNRS Éditions, 1999, $256 \mathrm{p}$.

Gunn, M. Désinsectiser les collections, la Lettre de l'OCIM, $\mathrm{n}^{\circ} 115$, 2008, pp. 15-22. 
Kingsley, H., Pinninger, D., Xavier-Rowe, A. et Winsor, P. Integrated pest management for collections. Proceding of 2001 : A Pest Odyssey, Londres : Éditions James \& James Ldt, 2001.

Michalsky, S. Normes de conservation : publication de nouvelles directives sur la température et l'humidité relative, Bulletin de l'ICC, n²4, novembre 1999.

Pinninger, D. Pest Management in museums, archives and historic houses. Londres : Éditions Archetype publications Ldt., 2004.

Rocquebert, M.-F. Les contaminants biologiques des biens culturels. Paris : Éditions Elsevier, 2002. 419 p.

Selwitz, C. et Maekava, S. Inert gases in control of museum pest. ÉtatsUnis : Editions The J. Paul Getty Trust,1998.

Site Internet pour consulter la norme NIMP15 : www.ippc.int

Strang, T. et Kigawa, R. La lutte contre les ravageurs des biens culturels, Bulletin technique de l'ICC, n²9, 2009, pp. 36-45.

Strang, T. Principle of heat treatment in Integrated pest management for collections. Proceding of 2001: A Pest Odyssey, pp. 114-129.
Thomson, R.-S. The effet of Thermo-Lignum Pest Eradication Treatment on Leather and other Skin Products. Rapport d'étude menée en 1995 au The Leather Consevation Centre, 34 Guildhal Road Northampton, NN1 lEW (communiqué par Thermo Lignum ${ }^{\circledR}$ ).

Thermo Lignum ${ }^{\circledR}$ Recherche sur le traitement thermique, contrôle de bois attaqué par des insectes nuisibles. Mémoire de diplôme de Fachhochschule (école technique supérieure) de Rosenheim, spécialité : technique du bois (Communiqué par Thermo Lignum ${ }^{\circledR}$ ).

Zycherman, L.-A. et Schrock, J.-R. A guide to museum pest control. Washington : Editions Foundations of American Institute for Conservation of Historic and Artistic Works and the Association of Systematic collections, 1998, $205 \mathrm{p}$ 